\title{
G

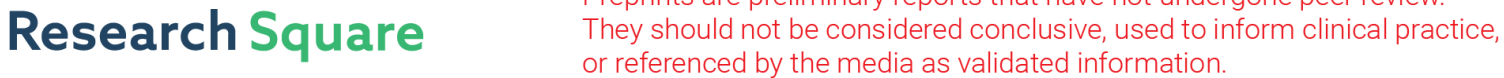

\section{Can Conventional MRI Features Predict H3K27M Mutation Status of Diffuse Midline Gliomas?}

Richa Singh Chauhan

NIMHANS: National Institute of Mental Health and Neuro Sciences

\section{Karthik Kulanthaivelu}

NIMHANS: National Institute of Mental Health and Neuro Sciences

Nihar Kathrani

Paras Hospitals

Abhishek Kotwal ( $\sim$ abhishekkotwal2009@gmail.com )

NIMHANS: National Institute of Mental Health and Neuro Sciences https://orcid.org/0000-0003-4308-

1704

Maya Dattatraya Bhat

NIMHANS: National Institute of Mental Health and Neuro Sciences

Jitender Saini

NIMHANS: National Institute of Mental Health and Neuro Sciences

Chandrajit Prasad

NIMHANS: National Institute of Mental Health and Neuro Sciences

Dhritiman Chakrabarti

NIMHANS: National Institute of Mental Health and Neuro Sciences

\section{Vani Santosh}

NIMHANS: National Institute of Mental Health and Neuro Sciences

Alok Mohan Uppar

NIMHANS: National Institute of Mental Health and Neuro Sciences

Dwarakanath Srinivas

NIMHANS: National Institute of Mental Health and Neuro Sciences

\section{Research Article}

Keywords: VASARI, H3K27M, diffuse midline glioma, MRI

Posted Date: March 31st, 2021

DOI: https://doi.org/10.21203/rs.3.rs-360529/v1

License: (9) (1) This work is licensed under a Creative Commons Attribution 4.0 International License.

Read Full License 
Page $2 / 20$ 


\section{Abstract}

Purpose Pre-surgical prediction of H3K27M mutation in diffuse midline gliomas (DMG) on MRI is desirable. The purpose of the study is to elaborate conventional MRI (cMRI) of H3K27M-mutant DMGs and identify features that could discriminate them from WT (wild type)-DMGs.

Methods cMRI features of 123 patients with DMG were evaluated conforming to the institutional research protocols. Multimodality MRI was performed on 1.5 or 3.0 Tesla MR Scanners with imaging protocol including T1w, T2w, FLAIR, diffusion-weighted, susceptibility-weighted and post- contrast T1w sequences. Pertinent cMRI features were annotated along the lines of Visually AcceSAble Rembrandt Images (VASARI) features and Intra Tumoral Susceptibility Signal score (ITSS) were evaluated. R software was used for statistical analysis.

Results Sixty-one DMGs were H3K27M-mutant (mutant DMGs). The patients in the H3K27M-mutant DMG group were younger compared to the WT-DMG group (WT DMGs) (mean age $24.13+13.13$ years vs. $35.79+18.74$ years $)(P=0.016)$. The two groups differed on $5 \mathrm{cMRI}$ features $-i)$ enhancement quality $(P=0.032)$, ii) thickness of enhancing margin $(P=0.05)$, iii) proportion of edema $(P=0.002)$, iv) definition of non-contrast enhancing tumor (NCET) margin $(P=0.001)$ and $v)$ cortical invasion $(P=0.037)$. The mutant DMGs showed greater enhancement and greater thickness of enhancing margin while the WT DMGs exhibited significantly larger edema proportion with poorly defined NCET margins and cortical invasion. ITSS was not significantly different among the groups.

Conclusion cMRI features like enhancement quality, thickness of the enhancing margin, proportion of edema, definition of NCET margin and cortical invasion can discriminate between the H3K27M-mutant and WT DMGs.

\section{Introduction}

Diffuse midline glioma, H3K27M-mutant, as a distinct set of tumors has been recently categorized in the revised 2016 World Health Organization (WHO) classification of the central nervous system (CNS) tumors that exploits an integrated diagnosis combining both histological features and molecular signature. The term assembles diffuse intrinsic pontine gliomas (DIPGs) and infiltrating high-grade glial tumors of the midline carrying the similar canonical mutation at the Lysine 27 residue of the $\mathrm{N}$-terminal tail of histone $\mathrm{H} 3$ with its unique recurrent substitution by methionine in the histone H3 variants, H3.3 (approximately $75 \%$ cases) and H3. 1 (approximately $25 \%$ cases). These histone variants are encoded by H3F3A and HIST1H3B/C genes, respectively [1-3]. This observation is in contrast to the hemispheric gliomas, where glycine to arginine substitution occurs in the H3F3A gene (H3G34R). The tumors harboring this mutation have been reported to be high-grade that clinically behave aggressively and portends an unfavorable outcome compared to their wild-type (WT) counterparts. Typically, these lesions display short median survival duration of approximately 9 to 11 months from the time of diagnosis regardless of the site of the tumor. They have, therefore, been designated by WHO as grade IV tumors irrespective of their histologic 
morphology [3-4]. Primary childhood brain neoplasms are rare lesions, with an incidence of $\sim 2,200$ cases per year and DIPG makes up $\sim 20 \%$ of these tumors [5]. Being a rare class of tumors, the exact incidence of DMGs harboring H3K27M mutation is still relatively unknown [6].

As opposed to the previous terminology of DIPG, the term "Diffuse Midline Glioma" (DMG) specifies that these lesions are not solely centered in the pons/ brainstem but may also originate in the other midline structures like thalami, gangliocapsular region, cerebellum, cerebellar peduncles, third ventricle, hypothalamus, and the pineal region as well as in the spinal cord [2,7-9]. Being a rare class of tumors, the exact incidence of DMGs harboring H3K27M mutation (mutant DMGs) remains uncertain. While historically recognized as a pediatric predilection, mutant DMGs can occur across all age groups $[6,10]$. Diffuse brainstem gliomas are, in general, more aggressive in the pediatric age group than in adults [2]. In children harboring mutant DMGs, the median survival of less than 12, with less than 10 percent 2-year survival rate, while some of the series of adult patients have shown variable median survival rates of up to 20 months $[5,6]$.

The critical anatomical location and the infiltrative nature of the tumor in the thalamus or brainstem limit any meaningful surgical resection, and treatment usually employed is fractionated radiotherapy (RT). Multiple monotherapy and combination chemotherapy regimens have also been tried with equivalently dismal outcomes [5]. Despite RT and chemotherapy, DMG in children had poor prognosis as a two-year overall survival rate of less than $10 \%$ [11]. Since the tumor involves eloquent areas of the brain, a biopsy is usually avoided, and diagnosis of DMGs hinges on clinical and imaging features [7,10]. Imaging modalities like computed tomography (CT) and magnetic resonance imaging (MRI) aid in localization, diagnosis, guiding treatment, and dynamically/ temporally monitoring the disease evolution and response to treatment. Conventional MRI (CMRI) is commonly used for brain tumor evaluation. The Visually AcceSAble Rembrandt Images (VASARI) MRI feature set has been designed to facilitate reliable depictions of gliomas through organized terminology and distinct visual imaging features. It is given by The Cancer Imaging Archive (TCIA) [12]. The use of the VASARI feature set has been adopted earlier for the molecular subtyping of gliomas and to predict IDH mutation status $[13,14]$. DMGs are frequently diagnosed based on CT or MRI features as part of these patients' initial evaluation. To the best of our knowledge, there are very few studies that has reported the use of conventional MRI features to differentiate the H3K27M mutant DMGs from their wild-type counterparts (WT DMGs). Further, none of the previous reports have utilized an extensive set of conventional imaging features used in the VASARI feature set for the distinction of mutational status in the DMGs.

Therefore, we studied the demographic, clinical, pathological, and imaging features of DMG patients retrospectively. We adopted the imaging features similar to those described in VASARI but relevant to midline gliomas for annotating these tumors on MRI. Our study aimed at assessing the cMRI markers to predict the H3K27M mutation status in DMGs non-invasively.

\section{Methods}


This was a retrospective observational study performed at a tertiary referral center conforming to the institutional research protocols. We searched our hospital's database retrospectively for the gliomas involving various midline structures of the brain, including the septal region, hypothalamus, thalamus brainstem, cerebellum, middle cerebellar peduncles, and pineal region from June 2016 to April 2020.

\section{Patient cohort}

The cases included in the study had, i) MRI suggestive of DMG, ii) who had not received any therapy or had not undergone biopsy or surgical resection before the MRI examination, and

iii) for whom the histopathology and immunohistochemistry (IHC) reports were available. All the patients underwent stereotactic biopsy or surgical resection within one month of the MRI. The exclusion criteria were: 1. patients with cerebral hemispheric mass lesions; 2 . patients with spinal cord lesions; 3 . those subjects where imaging or HPE was suggestive of WHO grade I pilocytic astrocytomas or tumor subtypes other than glioma; 4. inconclusive biopsy; 5 . biopsy-proven diffuse midline gliomas with no MRI study; and 6. immunohistochemistry for H3K27M mutation status unavailable. A total of 123 consecutive cases fulfilled the inclusion criteria and were finally enrolled in the study, of which 61 patients had mutant DMGs (age (years) $=24.13+13.13)$, and 62 patients had WT DMGs (age $=35.79+18.74)$ (in Online

\section{Resource 1).}

\section{Histopathology and Immunohistochemistry}

Ninety-seven of 123 patients underwent surgical resection, and 26 patients underwent stereotactic biopsy. All the tumors were reviewed by the Neuropathologist and were histologically categorized as phenotypic low-grade diffuse astrocytomas (grade II), anaplastic astrocytomas (grade III), glioblastomas (grade IV) and diffuse midline gliomas (grade IV). For IHC, formalin- fixed paraffin-embedded sections $(4 \mu \mathrm{m})$ from the blocks were collected on Silane coated slides. IHC was performed using the Ventana Benchmark automated staining system (Ventana Benchmark-XT). Briefly, the sections were subjected to antigen retrieval followed by incubation with primary and then secondary antibody. Counterstaining was done with hematoxylin. The antibodies used were as follows: H3K27me3 (Millipore, 07-449; 1:100) (H3.3K27Mme3, Malaysia, RM192, 1:100); Anti-mIDH1 R132H (dilution 1:50, internal clone H06, Dianova, Hamburg, Germany); Sigma polyclonal anti ATRX antibody in 1:100 dilutions; and DAKO P53 antibody in 1:200 dilutions, D07 clone. Appropriate positive or negative controls were included in each batch of staining. The neuroradiologists were blinded to the pathology results of the cases

\section{Image acquisition}

MRI was performed on the 1.5 (Aera 1.5 T, Siemens Medical Systems, Erlangen, Germany) or 3.0 (Achieva 3T, Philips Medical Systems, Best, Netherlands) Tesla MR Scanners using 32 channel head coil as per standard operating procedure with/without sedation. MRI protocol included the following sequences: T2weighted Turbo spin-echo axial and coronal (TR/TE: 3000-4900/80-99 ms), T1-weighted spin-echo axial (TR/TE: 500-600/10 ms), fluid- attenuated inversion recovery (FLAIR) (TR/TE: 9000-11000/ 87-125ms, IR 
delay- 2500-28003 ms) and SWI (susceptibility-weighted imaging)/ Venobold (TR/TE: 31-49/7.2-30 ms), prior to contrast administration with $512 \times 512$ matrix size, $5 \mathrm{~mm}$ slice thickness and $1 \mathrm{~mm}$ inter-slice gap. Post-contrast 3D T1 MPRAGE (Magnetization prepared rapid gradient echo) sequence was acquired after intravenous administration of gadolinium-based contrast agent (Gadovist (Gadobutrol)- $0.1 \mathrm{mmol} / \mathrm{kg}$ ): (256 x 256) matrix size, $1 \times 1 \times 1 \mathrm{~mm} 3$ in-plane resolution, TR/TE=6.7/3 ms (3T) and 2200/2.6 ms (1.5T). Intratumoral susceptibility signal (ITSS) scoring was also done on the Venobold and SWI images.

\section{Qualitative imaging features}

All the MRI scans were reviewed on picture archiving and communication system (PACS) by two independent neuroradiologists. Our analysis adopted the broad range of imaging attributes

as laid out in the VASARI dataset for a detailed characterization of the lesions on conventional MRI. The following parameters were evaluated: tumor location, side of lesion center, involvement of eloquent brain, enhancement quality, proportion contrast-enhancing tumor (CET), proportion non-contrast enhancing tumor (NCET), proportion necrosis, cysts, multifocal or multicentric or gliomatosis pattern, T1/FLAIR ratio, thickness of enhancing margin (maximum thickness), definition of the enhancing margin, definition of the non-enhancing margin, proportion of edema, hemorrhage, pial invasion, ependymal extension, cortical involvement, deep white matter invasion, NCET crosses midline, CET crosses midline and presence of satellites lesions as per the definition [12]. Few parameters like overall tumor margin and presence of exophytic component, hydrocephalus, and mass effect were also evaluated other than the VASARI feature set.

ITSS scoring was also performed as specified by Park et al. [15]. ITSS was defined as low signal tubular or dot-like structures with or without conglomeration within the tumor in high- resolution SWI. ITSS was divided into 4 grades: grade 0 (No ITSS); grade 1 (1-5 dot-like or tubular ITSS); grade 2 (6-10 dot-like or tubular ITSS), and grade 3 (> 11 dot-like or tubular ITSS).

Intergroup analysis of various features was done between overall mutant and WT DMGs irrespective of tumor grade and location. Furthermore, subgroup analysis for thalamic, brainstem, and grade IV mutant and WT DMGs was also performed.

\section{Statistical analysis}

Data was collated offline in a Microsoft Excel 2007 spreadsheet in a de-identified manner. The analysis was conducted using R software version 3.5.2. Interval scale data were presented as means and standard deviations, and nominal scale data are presented as frequencies and percentages. Between-group analysis of interval scale data was conducted using the non- parametric Mann-Whitney $U$ test. Normality of within-group data was observed qualitatively using histograms, and for conformity of analysis, nonparametric methods were chosen. Between-group analysis of nominal scale data was conducted using a Chi-square test with or without Yate's correction as appropriate. A P-value of $<0.05$ was considered statistically significant. 


\section{Results}

Sixty-one out of 123 cases (49.59\%) harbored H3K27M mutation, while 62 patients (50.41\%) had H3K27M WT status. The mean age of patients in the mutant DMG group was $24.13+13.13$ years, while in the WT group was $35.79+18.74$ years $(P=0.016)$. There were 62 female and 61 male patients, and gender distribution was not significantly different between the two groups. Mutant DMGs were graded as WHO grade IV tumors owing to positive H3K27M mutation status. Six (10\%) out of 62 WT DMGs were diffuse astrocytomas (grade II), 31 (50\%) were anaplastic astrocytomas (grade III), and 25 (40\%) were glioblastomas (grade IV).

In our study, the thalamus was the most favored site (64\%), followed by the brainstem (25\%) and other locations (11\%). Fifty-nine percent $(n=36)$ of the $61 \mathrm{H} 3 \mathrm{~K} 27 \mathrm{M}$ mutant tumors were located in the thalamus, $38 \%(n=23)$ in the brainstem $(13 \%, 21 \%$, and $3 \%$ in the midbrain, pons, and medulla, respectively), and the remaining $3 \%(n=2)$ were centered in the other locations (one each in the pineal region and cerebellum). Out of the 62 WT DMGs, $69 \%(n=43)$ were epicentered in the thalamus, $11 \%(n=7)$ in the brainstem $(3,6$, and 2 percent in the midbrain, pons, and medulla, respectively), and the rest $20 \%$ $(n=12)$ were located elsewhere; $3(5 \%)$ in septal region, $1(2 \%)$ in the pineal region, $6(9 \%)$ in middle cerebellar peduncles and $2(3 \%)$ in the cerebellum (Table 1). There was no statistically significant difference between the two groups based on the thalamic location $(P=0.313)$. However, the higher frequency of the brainstem location of mutant DMGs was significant $(P=0.0014)$.

On intergroup analysis between the mutant and WT DMGs, five of the studied conventional imaging features evaluated showed significant differences. These features were the $i$. enhancement quality $(P=0.032)$; ii. the thickness of the enhancing margin $(P=0.05)$; iii. proportion of edema $(P=0.002)$; iv. definition of NCET margin $(P=0.001)$ and v. cortical invasion $(P=0.037)$. The mutant $D M G s$ showed significantly greater enhancement as well as the greater thickness of enhancing margin or solid enhancement. The WT DMGs exhibited a significantly larger proportion of edema with a poorly defined NCET margin and a larger degree of cortical invasion compared with the mutant DMGs (in Fig. 1-3). Few other parameters like tumor heterogeneity $(P=0.07)$, overall tumor margin $(P=0.074)$, and definition of CET margin $(P=0.073)$ tend to approach the level of significance with a higher trend of heterogeneity in the mutant group, and WT tumors were showing more ill-defined margins (Table 2).

On subgroup analysis, an additional feature of the presence of exophytic component showed significant difference with mutant thalamic gliomas (52.78\%) showing more exophytic component as compared to the WT gliomas (23.25\%) ( $P=0.013$ ) (in Fig. 3). Brainstem tumors did not show any difference in terms of the presence of edema.

Twenty-seven (47.4\%) of the mutant tumors showed ITSS grade 0, 1 (1.8\%) ITSS grade 1, 2 (3.5\%) ITSS grade 2 and 27\% (47.4\%) ITSS grade 3; whereas 25 (44.6\%) WT tumors exhibited ITSS grade 0,7 (12.5\%) ITSS grade $1,3(5.4 \%)$ ITSS grade 2 and $21(37.5 \%)$ showed ITSS grade 3 . ITSS score was not found to be significantly different between mutant and WT DMGs $(P=0.681)$. 


\section{Discussion}

As per the decision of Working Committee 3 of the cIMPACT-NOW, the term DMG, H3K27M-mutant should be earmarked for the tumors that are diffuse, i.e., infiltrating, midline (e.g., involving the thalamus, brain stem, spinal cord, etc.), gliomas (astrocytic lineage) and H3K27M mutant, and must not be used for other tumors (as ependymoma or pilocytic astrocytoma) that possess H3K27M mutation [16]. H3K27M mutation is a gain-of-function mutation that modifies gene expression by posttranslational alterations in histone three, leading to altered DNA methylation and gliomagenesis via epigenetic regulation [4]. Genomic analysis of mutant DMGs has shown many correlated genetic variations, including mutations in the receptor tyrosine kinase/RAS/PI3K pathway, p53 overexpression, and ATRX (alphathalassemia/mental retardation syndrome X-linked) loss [1,9].

In this study, approximately half of the midline located gliomas harbored the H3K27M mutation and were designated as DMG, H3K27M-mutant. Such incidence of the H3K27M mutation in midline gliomas has also been previously reported $[10,17,18]$. Mutant DMGs occur in the younger population as compared to WT DMGs [10]. In our study, the patients with mutated DMGs were approximately 12 years younger than WT DMG patients $(P=0.016)$. We did not find any significant difference in the gender distribution between the mutant and WT groups. The same has been observed earlier in the studies by Chen et al. and Schreck et al. $[6,10]$.

We found that most of the DMGs $(79 \%)$ were centered in the thalamus (with no significant difference between the groups). However, the difference was significant when brainstem location was considered, and a greater number of mutant DMGs were distributed in the brainstem than WT-DMGs. A similar finding has been reported by Schreck et al. [6].

Our results revealed that certain cMRI features could be used to discriminate the H3K27M mutational status of DMGs. On intergroup and subgroup (brainstem and grade IV tumors) analyses, we found that the mutant gliomas demonstrated greater enhancement with thicker enhancing margins or solid enhancement as compared to the WT group. In comparison, the WT tumors exhibited more edema, poorly defined NCET margins, and more cortical invasion compared with the mutant DMGs. In the radiological evaluation results in the HERBY Phase II trial of non-brainstem pediatric HGGs, the mutated DMGs have been reported to enhance avidly compared to WT DMGs [19]. The H3K27M mutation escalates the expression of growth factors like PDGF and VEGF by recurrent mutations and amplification of signaling genes like ACVR1, PI3K, and RTKs. The PIK3R1 mutations are reported to be obligatory to H3.3K27M mutations, and PIK3CA mutations are found in all DIPGs. These PIK3 mutations are the promoters of angiogenesis [20-21] that might account for the more florid enhancement of the mutant gliomas that we appreciated in this study.

Histopathology literature is found wanting as to why mutant DMGs demonstrated a lesser degree of edema compared with the WT tumors. However, the same finding has also been discussed by Qui et al. in their study of 66 patients with DMGs, where only 10 cases showed peritumoral edema [22]. Castel et al. have reported that the more aggressive H3.3mutant DMGs exhibited lesser extracellular edema as 
compared to the less aggressive H3.1 mutant DMGs [23]. On the other hand, some authors found no significantly different cMRI parameters (such as tumor location, margin, cysts, necrosis, hemorrhage, degree, and pattern of contrast enhancement and edema) that could distinguish the mutant from WT DMGs $[2,6,10,23,24]$.

When we compared the cMRI features between thalamic mutant and WT tumors, mutant tumors showed more exophytic components. WT tumors showed significantly more edema; however, there was no difference in the enhancement and the definition of tumor margin between the two groups. The presence of exophytic growth pattern (tumor growing outside the borders of the structure of origin) confers a superior prognosis in brainstem gliomas than patients with tumors showing intrinsic growth patterns [25-27]. However, the same has not been described for the thalamic gliomas. This could indicate that the influence of this mutation in different anatomical locations may be variable, and these tumor subsets can be studied in a more comprehensive manner.

The limitations of this study include the lack of follow up and no information regarding the survival data of the patients. Also, we did not perform any measurements to quantitate the degree of enhancement, which could increase the reproducibility of the results. Subgrouping of the histone of the H3.3/H3.1 gene group that could have been interesting was not performed in our study. Future studies with more extensive clinical data are needed to recognize various other aspects of H3K27M mutant DMGs, like outcome and management strategies, which are not discussed in this paper.

\section{Conclusion}

Conventional MRI features such as enhancement quality, the thickness of the enhancing margin, proportion of edema, definition of non-contrast enhancing tumor margin, and cortical invasion can help discriminate between the H3K27M-mutant and WT DMGs. Among the varied imaging phenotypic characteristics of DMGs, these findings carry an important implication for treatment planning as well as designing future trials in this distinct group of neoplasms.

\section{Declarations}

\section{Acknowledgments}

Not applicable.

\section{Funding}

No funding was received for conducting this study.

\section{Conflicts of interest/Competing interests}

The authors have no relevant financial or non-financial interests to disclose. 
Availability of data and material (data transparency)

The datasets generated during and/or analysed during the current study are available from the corresponding author on reasonable request.

\section{Code availability}

The datasets generated during and/or analysed during the current study are available from the corresponding author on reasonable request.

\section{Authors' contributions}

All authors contributed to the study conception and design. Material preparation, data collection and analysis were performed by Richa Singh Chauhan, Maya Dattatraya Bhat and Karthik Kulanthaivelu. The first draft of the manuscript was written by Richa Singh Chauhan and all authors commented on previous versions of the manuscript. All authors read and approved the final manuscript.

\section{Ethics approval}

All procedures performed in studies involving human participants were in accordance with the ethical standards of the institutional research committee and with the 1964 Helsinki Declaration and its later amendments or comparable ethical standards. The study was approved by the Bioethics Committee of the National institute of mental health and neurosciences (NIMHANS), Bengaluru, India.

\section{Consent to participate}

Informed consent was obtained from all individual participants included in the study.

\section{Consent for publication}

Patients signed informed consent regarding publishing their data and photographs.

\section{References}

1. Wang L, Li Z, Zhang M, Piao Y, Chen L, Liang H, et al. H3 K27M-mutant diffuse midline gliomas in different anatomical locations. Hum Pathol. 2018;78:89-96.

2. Daoud E V., Rajaram V, Cai C, Oberle RJ, Martin GR, Raisanen JM, et al. Adult brainstem gliomas with H3K27M mutation: Radiology, pathology, and prognosis. J Neuropathol Exp Neurol. 2018;77(4):30211.

3. Louis DN, Ohgaki H, Wiestler OD, Cavenee WK, Ellison DW, Figarella-Branger D, et al. (2016) The 2016 WHO classification of tumors of the central nervous system. International Agency for Research on Cancer (IARC), Lyon. 
4. Khuong-Quang DA, Buczkowicz P, Rakopoulos P, Liu XY, Fontebasso AM, Bouffet E, et al. K27M mutation in histone $\mathrm{H} 3.3$ defines clinically and biologically distinct subgroups of pediatric diffuse intrinsic pontine gliomas. Acta Neuropathol. 2012;124(3):439-47.

5. Himes BT, Zhang L, Daniels DJ. Treatment strategies in diffuse midline gliomas with the H3K27M mutation: The role of convection-enhanced delivery in overcoming anatomic challenges. Front Oncol. 2019;9(FEB):1-10.

6. Schreck KC, Ranjan S, Skorupan N, Bettegowda C, Eberhart CG, Ames HM, et al. Incidence and clinicopathologic features of $\mathrm{H} 3 \mathrm{~K} 27 \mathrm{M}$ mutations in adults with radiographically-determined midline gliomas. J Neurooncol. 2019;143(1):87-93.

7. Johnson DR, Guerin JB, Giannini C, Morris JM, Eckel LJ, Kaufmann TJ. 2016 updates to the WHO brain tumor classification system: What the radiologist needs to know. Radiographics. 2017;37(7):2164-80.

8. Louis DN, Perry A, Reifenberger G, von Deimling A, Figarella-Branger D, Cavenee WK, et al. The 2016 World Health Organization Classification of Tumors of the Central Nervous System: a summary. Acta Neuropathol. 2016;131(6):803-20.

9. Solomon DA, Wood MD, Tihan T, Bollen AW, Gupta N, Phillips JJJ, et al. Diffuse Midline Gliomas with Histone H3-K27M Mutation: A Series of 47 Cases Assessing the Spectrum of Morphologic Variation and Associated Genetic Alterations. Brain Pathol. 2016;26(5):569-80.

10. Chen H, Hu W, He H, Yang Y, Wen G, Lv X. Noninvasive assessment of H3 K27M mutational status in diffuse midline gliomas by using apparent diffusion coefficient measurements. Eur $\mathrm{J}$ Radiol. 2019;114:152-9.

11. Seong M, Kim ST, Noh JH, Kim YK, Kim HJ. Radiologic findings and the molecular expression profile of diffuse midline glioma H3 K27M mutant. Acta radiol. 2020;Nov11:284185120968560.

12. Jarosz Qon (2020) VASARI Research Project - The Cancer Imaging Archive (TCIA) Public Access Cancer Imaging Archive Wiki. Cancer Imaging Archive Wiki. https:// wiki.cancerimagingarchive.net/display/Public/VASARI/Research/Project. Accessed 20 Mar. 2021.

13. Zhou J, Reddy M V., Wilson BKJ, Blair DA, Taha A, Frampton CM, et al. MR Imaging Characteristics Associate with Tumor-Associated Macrophages in Glioblastoma and Provide an Improved Signature for Survival Prognostication. Am J Neuroradiol. 2018;39(2):252-259.

14. Zhou $H$, Vallières $M$, Bai $H X$, Su $C$, Tang $H$, Oldridge $D$, et al. MRI features predict survival and molecular markers in diffuse lower-grade gliomas. Neuro Oncol. 2017;19(6):862-70.

15. Park SM, Kim HS, Jahng GH, Ryu CW, Kim SY. Combination of high-resolution susceptibility-weighted imaging and the apparent diffusion coefficient: Added value to brain tumour imaging and clinical feasibility of non-contrast MRI at $3 \mathrm{~T}$. Br J Radiol. 2010;83(990):466-75.

16. Louis DN, Giannini C, Capper D, Paulus W, Figarella-Branger D, Lopes MB, et al. cIMPACT-NOW update 2: diagnostic clarifications for diffuse midline glioma, H3 K27M- mutant and diffuse astrocytoma/anaplastic astrocytoma, IDH-mutant. Acta Neuropathol. 2018;135(4):639-42. 
17. Feng J, Hao S, Pan C, Wang Y, Wu Z, Zhang J, et al. The H3.3 K27M mutation results in a poorer prognosis in brainstem gliomas than thalamic gliomas in adults. Hum Pathol. 2015;46:1626-32.

18. Buczkowicz P, Bartels U, Bouffet E, Becher O, Hawkins C. Histopathological spectrum of paediatric diffuse intrinsic pontine glioma: diagnostic and therapeutic implications. Acta Neuropathol. 2014;128(4):573-81.

19. Gutierrez DR, Jones C, Varlet P, Mackay A, Warren D, Warmuth-Metz M, et al. Radiological evaluation of newly diagnosed non-brainstem pediatric high-grade glioma in the herby phase II trial. Clin Cancer Res. 2020;26(8):1856-65.

20. Duchatel RJ, Jackson ER, Alvaro F, Nixon B, Hondermarck H, Dun MD. Signal Transduction in Diffuse Intrinsic Pontine Glioma. Proteomics. 2019;19:e1800479.

21. Mazur M, Couldwell W. Investigating a Candidate Cell of Origin for Diffuse Intrinsic Pontine Glioma. World Neurosurg. 2011;76(5):368-369.

22. Qiu T, Chanchotisatien A, Qin Z, Wu J, Du Z, Zhang X, et al. Imaging characteristics of adult H3 K27M-mutant gliomas. J Neurosurg. 2019;Nov 15:1-9.

23. Castel D, Philippe C, Calmon R, Le Dret L, Truffaux N, Boddaert N, et al. Histone H3F3A and HIST1H3B K27M mutations define two subgroups of diffuse intrinsic pontine gliomas with different prognosis and phenotypes. Acta Neuropathol. 2015;130(6):815-27.

24. Makepeace L, Scoggins M, Mitrea B, Li Y, Edwards A, Tinkle CL, et al. MRI patterns of extrapontine lesion extension in diffuse intrinsic pontine gliomas. Am J Neuroradiol. 2020;41(2):323-30.

25. Yoshikawa A, Nakada M, Watanabe T, Hayashi Y, Sabit H, Kato Y, et al. Progressive adult primary glioblastoma in the medulla oblongata with an unmethylated MGMT promoter and without an IDH mutation. Brain Tumor Pathol. 2013;30:175-9.

26. Cohen ME, Duffner PK, Heffner RR, Lacey DJ, Brecher M. Prognostic factors in brainstem gliomas. Neurology. 1986;36:602-5.

27. Horita Y, Wanibuchi M, Akiyama Y, Suzuki K, Omori Y, lihoshi S et al. Exophytic glioblastoma multiforme originating from the medulla oblongata. Biomed Research and Clin Practice. 2016;1(2):58-61.

\section{Tables}

Table 1 Patients' demographic data and location of the mutant and WT DMGs 


\begin{tabular}{|c|c|c|c|}
\hline & All patients $(n=123)$ & H3K27M Mutant $(n=61)$ & H3K27M WT $(n=62)$ \\
\hline Age range (in years) & $2-72$ & $3-55$ & $2-72$ \\
\hline Mean \pm SD & $30.01+17.16$ & $24.13+13.13$ & $35.79+18.74$ \\
\hline \multicolumn{4}{|l|}{ Gender } \\
\hline \multirow[t]{2}{*}{ Male Female } & 61 & 28 & 33 \\
\hline & 62 & 33 & 29 \\
\hline \multicolumn{4}{|l|}{ Location (n (\%)) } \\
\hline Thalamus & $79(64)$ & $36(59)$ & $43(69)$ \\
\hline Midbrain & $10(8)$ & $8(13)$ & $2(3)$ \\
\hline Pons & $17(14)$ & $13(21)$ & $4(6)$ \\
\hline Medulla & $3(3)$ & $2(3)$ & $1(2)$ \\
\hline Others & $14(11)$ & $2(3)$ & $12(20)$ \\
\hline
\end{tabular}

Table 2 The conventional imaging features of mutant and WT DMGs, irrespective of tumor grade and location, which showed significant difference (or tend to approach the level of significance) 


\begin{tabular}{|c|c|c|c|c|}
\hline MRI Features & & $\begin{array}{l}\text { Mutant, N } \\
(\%)\end{array}$ & $\begin{array}{l}\text { Wild-type, N } \\
\text { (\%) }\end{array}$ & $\begin{array}{l}\mathrm{P} \text { - } \\
\text { value }\end{array}$ \\
\hline \multirow[t]{3}{*}{ Enhancement quality } & None & $9(14.75)$ & $20(32.26)$ & 0.032 \\
\hline & Minimal/mild & $8(13.11)$ & $11(17.74)$ & \\
\hline & Marked/avid & $44(72.13)$ & $31(50)$ & \\
\hline \multirow{4}{*}{$\begin{array}{l}\text { Thickness of enhancing } \\
\text { margin }\end{array}$} & NA & $9(14.75)$ & $20(32.26)$ & 0.050 \\
\hline & Thin/ <3 mm & $23(37.7)$ & $13(20.97)$ & \\
\hline & Thick/ > 3 mm & $9(14.75)$ & $6(9.68)$ & \\
\hline & Solid & $20(32.79)$ & $23(37.1)$ & \\
\hline \multirow[t]{4}{*}{ Definition of NCET margin } & NA & $0(0)$ & $2(3.23)$ & 0.001 \\
\hline & well defined & $29(47.54)$ & $12(19.35)$ & \\
\hline & Equivocal & $11(18.03)$ & $8(12.9)$ & \\
\hline & Poorly-defined & $21(34.43)$ & $40(64.52)$ & \\
\hline \multirow[t]{4}{*}{ Proportion of edema } & None/ 0 & $26(42.62)$ & $13(20.97)$ & 0.002 \\
\hline & $<5 \%$ & $23(37.7)$ & $18(29.03)$ & \\
\hline & $6-33 \%$ & $9(14.75)$ & $16(25.81)$ & \\
\hline & $34-67 \%$ & $3(4.92)$ & $15(24.19)$ & \\
\hline \multirow[t]{2}{*}{ Cortical invasion } & No & $48(78.69)$ & 37 (59.68) & 0.037 \\
\hline & Yes & $13(21.31)$ & $25(40.32)$ & \\
\hline \multirow[t]{2}{*}{ Heterogeneity } & Homogeneous & $11(18.03)$ & $16(25.81)$ & 0.07 \\
\hline & $\begin{array}{l}\text { Mostly } \\
\text { homogeneous }\end{array}$ & $25(40.98)$ & $12(19.35)$ & \\
\hline
\end{tabular}




\begin{tabular}{|lllll|}
\hline & Mixed & $14(22.95)$ & $17(27.42)$ & \\
& Mostly & $11(18.03)$ & $17(27.42)$ & \\
& heterogeneous & & & \\
\hline Tumor margin & Ill-defined & $18(29.51)$ & $29(46.77)$ & 0.074 \\
\hline & Well-defined & $43(70.49)$ & $33(53.23)$ & \\
\hline $\begin{array}{l}\text { Definition of CET } \\
\text { margin }\end{array}$ & NA & $9(14.75)$ & $20(32.26)$ & 0.073 \\
& & & & \\
\hline & Well-defined & $27(44.26)$ & $20(32.26)$ & \\
\hline & Equivocal & $5(8.2)$ & $8(12.9)$ & \\
\hline III-defined & $20(32.79)$ & $14(22.58)$ & \\
\hline
\end{tabular}

Figures 

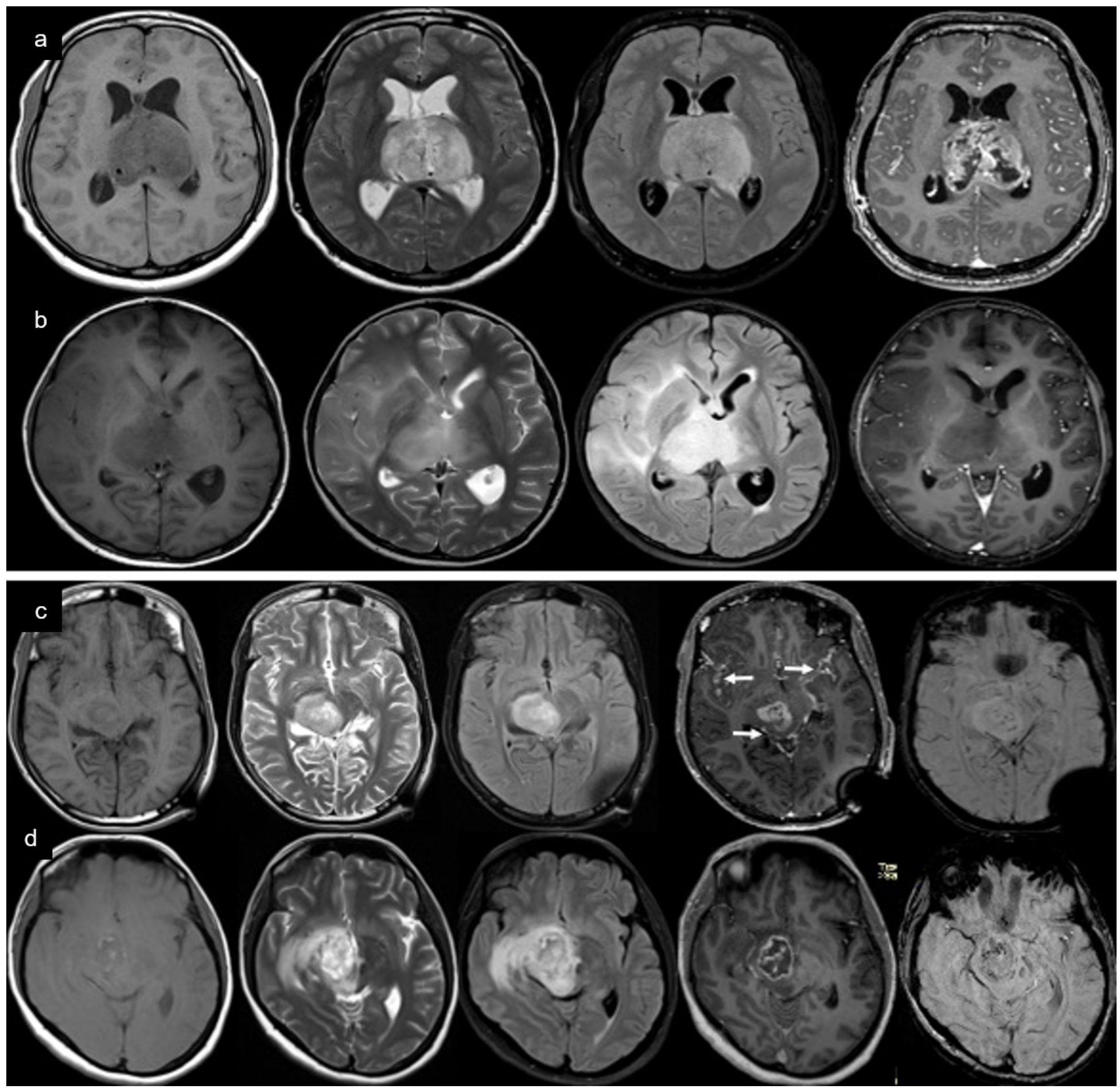

Figure 1

Examples of H3K27M-mutant and wild-type Bithalamic ( $a$ and b) and Midbrain ( $c$ and d) diffuse midline gliomas. A 24-year male patient with H3K27M-mutant DMG (a, first row) shows a well-marginated, heterogeneous bithalamic lesion with no associated edema, intense post contrast enhancement with thick enhancing margins. A 9-year male child with H3K27M wild-type DMG (b, second row) shows a homogeneous bithalamic tumor with extensive edema in right cerebral hemisphere, poorly-marginated non-contrast enhancing tumor and no post contrast enhancement. A 17-year male with H3K27M-mutant (c, third row) DMG shows a heterogeneous lesion without perilesional edema, solid enhancement with 
well-marginated non-contrast enhancing tumor. There is pial extension of the lesion with leptomeningeal enhancement along the ambient cistern and bilateral sylvian fissures (arrows). Note that the lesion shows ITSS of 3. A 45-year male with H3K27M wild-type DMG (d, last row) shows a heterogeneous lesion with associated perilesional edema, poor margins of the NCET and thin rim enhancement. Note the intralesional ITSS of 3
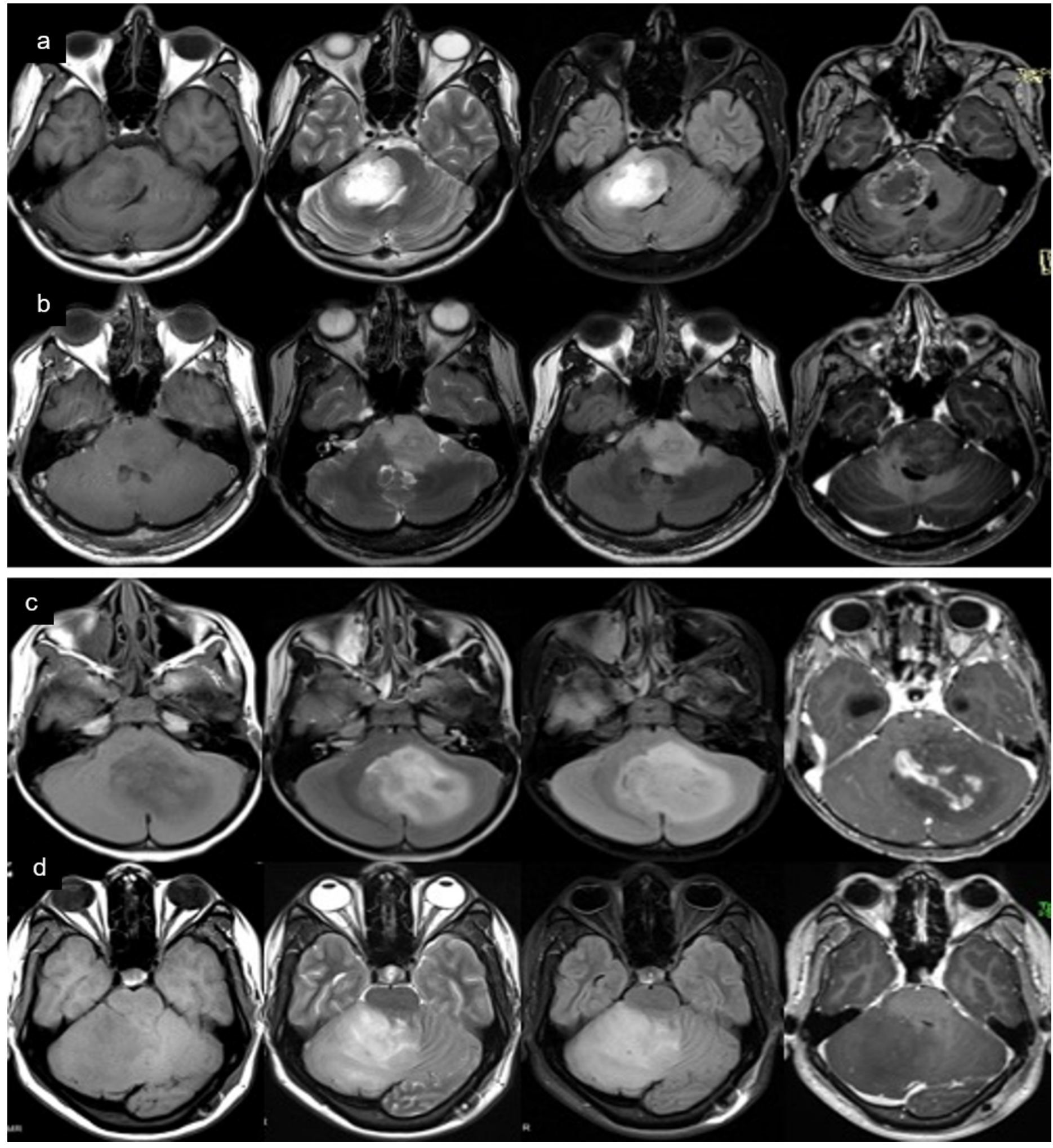

Figure 2 
Examples of H3K27M-mutant and wild-type DMGs involving the middle cerebellar peduncle (MCP) and pons ( $a$ and $b$ ) and cerebellum ( $c$ and d). A 17-year male with H3K27M- mutant ( $a$, first row) DMG shows a well-defined, heterogeneous tumor involving right hemipons and MCP without edema and thick enhancing margin. A 34-year male with H3K27M wild-type DMG (b, second row) shows a poorlymarginated tumor involving left hemipons and MCP with no associated edema and no post contrast enhancement. A 6-year female child with H3K27M- mutant (c, third row) DMG shows a well-marginated, mildly heterogeneous tumor involving left cerebellar hemisphere with no perilesional edema. There is patchy solid intense post contrast enhancement within the tumor. A 23-year male with H3K27M wild-type DMG (d, last row) shows a poorly-marginated tumor involving right cerebellar hemisphere, mild perilesional associated edema and no post contrast enhancement 


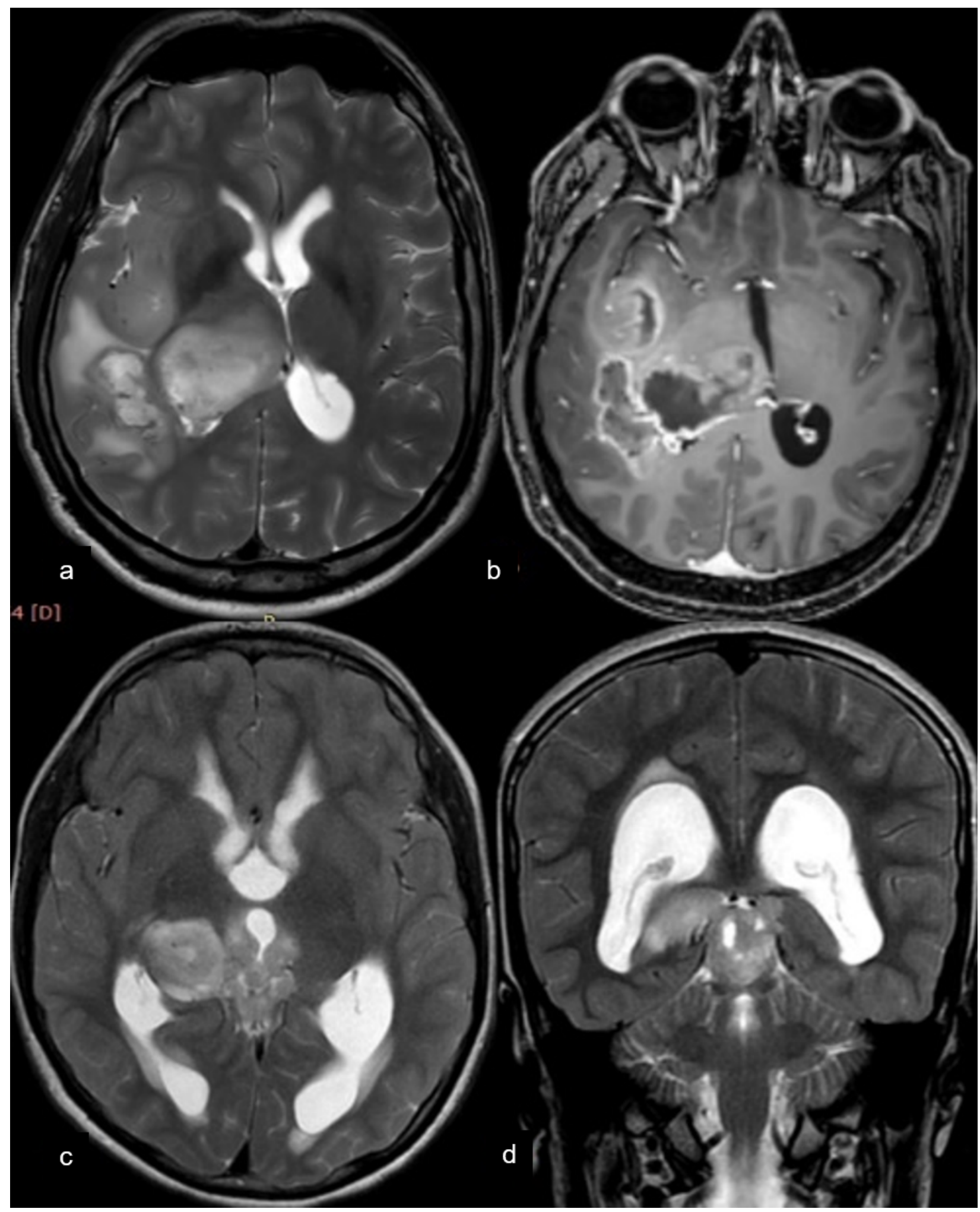

Figure 3

A 38-year male with H3K27M wild-type right thalamic glioma (a, upper row). The lesion shows perilesional edema, cortical invasion and thin post contrast rim enhancement. Another 18-year male patient with H3K27M-mutant (b, lower row) right thalamic glioma. The lesion shows well-defined margins, no significant perilesional edema and exophytic component extending into the superior cerebellar cistern 


\section{Supplementary Files}

This is a list of supplementary files associated with this preprint. Click to download.

- SupplementaryInformationSI.docx 